\title{
Protocol
}

\section{Assay for Courtship Suppression in Drosophila}

\author{
Aki Ejima and Leslie C. Griffith
}

\section{INTRODUCTION}

Unsuccessful courtship reduces subsequent courtship behavior of male Drosophila. This experiencedependent behavior modification is called courtship conditioning and has been one of the major paradigms used to study learning and memory in Drosophila. This article describes a basic behavioral assay for quantifying short-term memory of courtship suppression induced by training with a mated female.

\section{RELATED INFORMATION}

The quantification of courtship behaviors is covered in detail in Measurement of Courtship Behavior in Drosophila melanogaster (Ejima and Griffith 2007).

\section{MATERIALS}

CAUTIONS AND RECIPES: Please see the end of this protocol for appropriate handling of materials marked with $\langle$ ! $>$, and recipes for reagents marked with $<\mathbf{R}\rangle$.

\section{Reagents}

$<!>\mathrm{CO}_{2}$

$<!>$ Ether can be used as an alternative (see Step 6).

Drosophila melanogaster, naive males and females (wild-type; e.g., Canton-S or Oregon-R)

$<$ R $>$ Food media

\section{Equipment}

Aspirator, mouth (see Fig. 1)

Bunsen burner (for mouth aspirator; see Fig. 1)

Courtship chamber

A dissection plate plus slide glass (Fig. 1B) can be used as a courtship chamber. Slide the slide glass lid on the dissection plate and introduce animals through the gap into the hollow. A wheel chamber made with Plexiglas is suitable for observation of multiple animals. Rotate the top layer and introduce animals through an entrance hole into each cell (see Movie 1).

Depression slide, 3-well, deep

Filter paper, e.g. Whatman ashless no. 42

Place water-soaked filter paper in each cell of the courtship chamber.

Incubator preset to $25^{\circ} \mathrm{C}$, with a light/dark (L/D) cycle

Light source, white (for bright conditions) or dim-red (>700 nm, for dark conditions; see Discussion) 


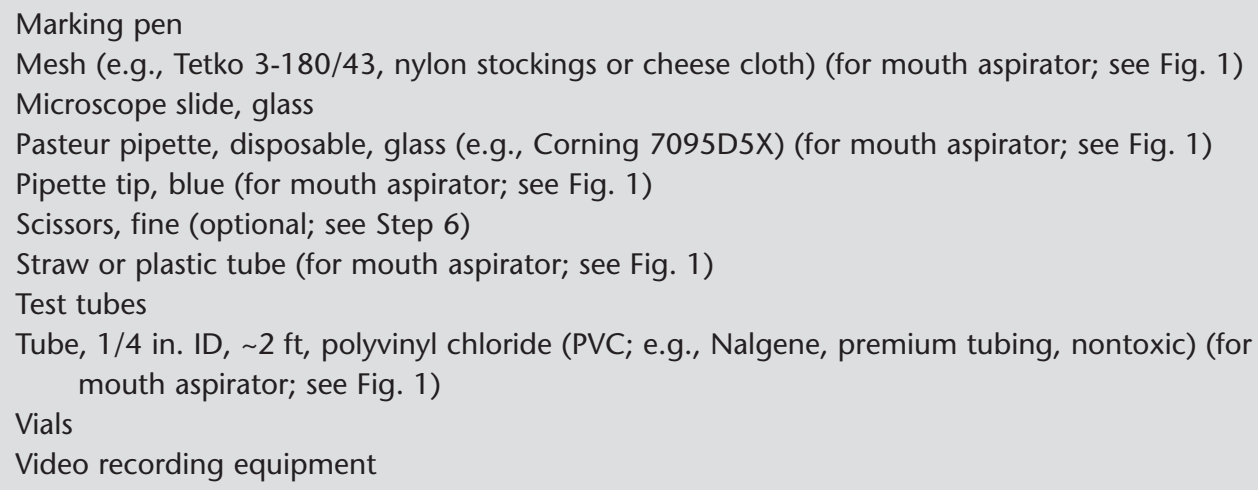

\section{METHOD}

\section{Preparing Adult Flies}

1. Raise flies at $25^{\circ} \mathrm{C}$ and $70 \%$ relative humidity under a 12-h/12-h L/D cycle.

2. Collect the flies on the day of eclosion and keep them in a $25^{\circ} \mathrm{C}$ incubator with an L/D cycle. Use autoclaved food with no sprinkled yeast granules (to avoid ethanol exposure).

i. Collect naive males and keep them in individual test tubes (to prevent pretest social experience).

ii. Collect target females and males for mating and store in groups (e.g., 30 animals per vial).

3. Maintain the collected flies for 4-5 d until they are sexually mature.

\section{Preparing Mated Female Trainers}

4. On the day before courtship training, pair a virgin female and a male (both group-stored, 3- or 4-d-old) and wait for copulation.

5. Collect and store the mated females whose copulations lasted longer than 14 min (Canton-S). The average "full-copulation" duration is variable, depending on the genetic background. Determine the cutoff duration by checking fertility and remating rates in advance.

\section{Courtship Training and Test}

6. To prepare immobilized tester females, anesthetize them with $\mathrm{CO}_{2}$ or ether, and freeze-kill or decapitate them with fine scissors immediately before the experiment. Mild $\mathrm{CO}_{2}$ exposure for 50 min causes immobilization for the following $10 \mathrm{~min}$. Apply strong ether vapor to kill the females. Some investigators prefer to avoid the use of ether, because ether fumes may be emitted by the female.

7. Assemble a courtship chamber containing wet filter paper in each cell to maintain humidity.

8. Label the chamber with the date and fly identification numbers only. Do not include genotype or specific manipulation.

9. Transfer a test male (individual-stored, naive) to each cell of the chamber using a mouth aspirator without anesthesia (see Movie 1)

10. Allow the males to acclimate to the chamber for $5 \mathrm{~min}$.

11. Using a mouth aspirator, introduce a trainer female (live and mated the day before) to each cell and immediately start video recording for $10 \mathrm{~min}$ (initial courtship, Fig. 2). 

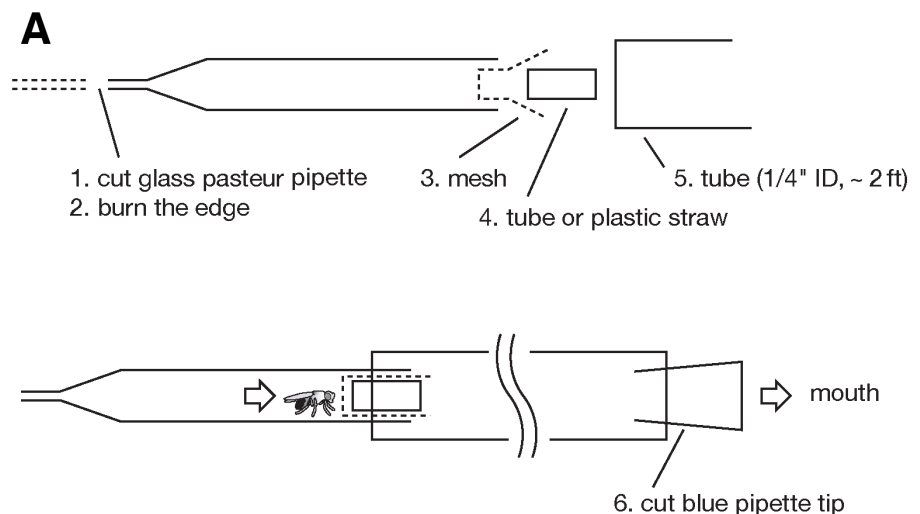

B

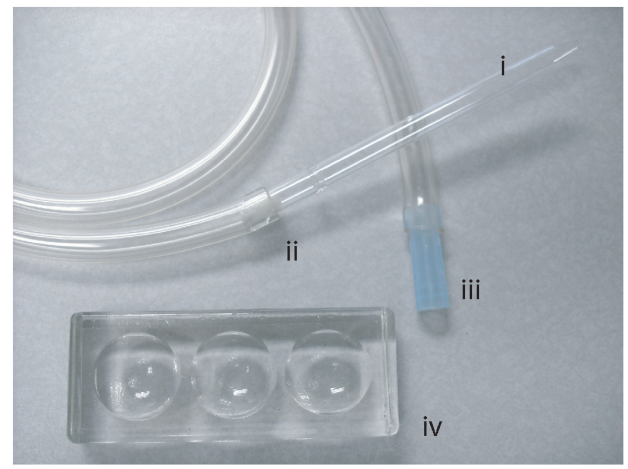

FIGURE 1. (A) Constructing a mouth aspirator. (1) Cut the tip of a glass Pasteur pipette. (2) Burn the tip in a Bunsen burner to heat-polish the sharp cutting edge and adjust the hole size. (3) Insert a piece of mesh cloth. (4) Insert a piece of plastic tube or straw to hold the mesh against the inside wall of the Pasteur pipette. (5) Cover the tail end of the glass Pasteur pipette with 1/4 in. ID PVC tube. (6) Cut tapered tip of a blue pipette tip (mouthpiece) and insert it into the tail end of tube. A fly aspirated into the Pasteur pipette will be trapped in front of the mesh. Arrows represent airflow on suction. (B) Photo of a mouth aspirator and a dissection-plate chamber. (i) Pasteur pipette; (ii) mesh trap; (iii) mouthpart; (iv) dissection plate and slide glass.

12. Observe the pair together for a total of $60 \mathrm{~min}$. Video record the last $10 \mathrm{~min}$ of the $60 \mathrm{~min}$ training (final courtship).

13. Transfer the trained male to a clean courtship chamber. Add an immobilized virgin tester female (from Step 6) immediately after training (0-min memory) or after a certain period of time (1-3 h, short-term memory) and video record the first 10 min (test courtship).

14. As a sham-trained control, isolate a male in a courtship chamber for $60 \mathrm{~min}$ and then test for courtship toward the tester female.

\section{Analysis of Video Recordings}

15. Analyze the video recordings.

During the behavioral analysis, the observer should refer to only the fly identification numbers and must be blind to the other experimental information (e.g., genotype or specific manipulation).

16. For each 10-min recording, calculate courtship index $(\mathrm{Cl}), \mathrm{Cl}_{\text {initial' }} \mathrm{Cl}_{\text {final' }^{\prime}} \mathrm{Cl}_{\text {test' }}$ and $\mathrm{Cl}_{\text {sham }}$, which is the fraction of time a male spent in courtship activity during the 10 -min observation period (see Measurement of Courtship Behavior in Drosophila melanogaster [Ejima and Griffith 2007]).

If pairs copulate during the training period or when male flies show low $\mathrm{Cl}_{\text {initial }}(<0.1)$, then the data are eliminated from future analysis.

17. Determine the learning index, which is the ratio of the courtship level in the final 10 min of the training $\left(\mathrm{Cl}_{\text {final }}\right)$ to that of the initial $10 \mathrm{~min}\left(\mathrm{Cl}_{\text {initial }}\right)$.

This shows how the courtship level changed during training (Fig. 2B). 


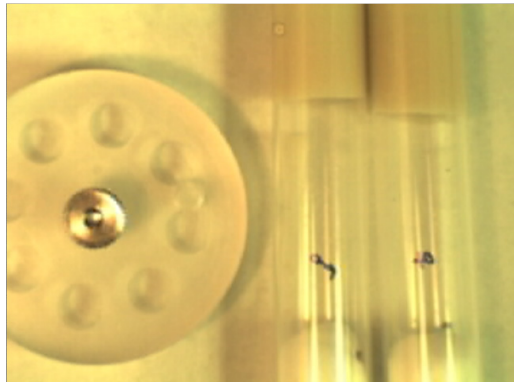

MOVIE 1. Introduction of flies into a wheel chamber using a mouth aspirator. (To view movie, see doi: 10.1101/pdb.prot5575 online at www. cshprotocols.org.)

18. Calculate the memory index by dividing $\mathrm{Cl}_{\text {test }}$ by the mean of the sham control courtship levels $\left(\mathrm{Cl}_{\text {sham }}\right)$.

A memory index $\approx 1$ indicates that there is no memory because the courtship level of the trained males is equivalent to that of the sham-trained males.

\section{DISCUSSION}

The courtship conditioning protocol we described in this article is the most conventional one used and involves a mated female trainer and a virgin female tester. Courtship memory produced by this protocol is considered to be associative, but it requires active performance of the male during training for effective modification (Ackerman and Siegel 1986; Ejima et al. 2005). A potentially damaged or sick male that has shown low courtship at the initial recording is considered to lack the training experi-

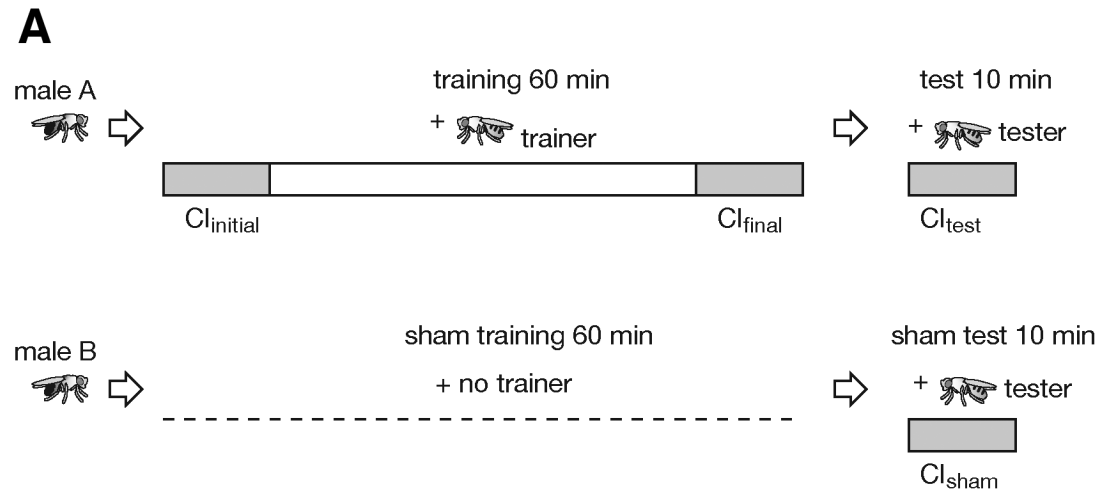

\section{B}
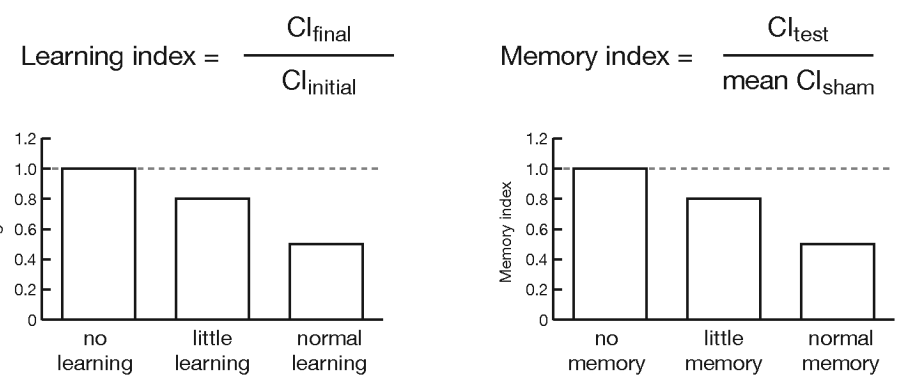

FIGURE 2. Schema of the courtship training and testing process. (A) Male A is paired with a trainer female for 60 min and the courtship response is video recorded during the first and last $10 \mathrm{~min}$ (shadowed boxes). After the training, the trained male is transferred to a clean chamber and paired with a tester female (test courtship). A sham control (male B) is kept alone in a chamber and then receives the same test (sham test). $(B)$ Representative learning and memory indices. If the courtship level in the final 10 min of training is equivalent to that of the initial $10 \mathrm{~min}$ (learning index $\approx 1.0$ ), the male did not reduce the courtship intensity during the training period. A memory index $\approx 1.0$ means that courtship after training is at the same level as sham, indicating that there was no behavioral modification by the training. (Cl) Courtship index. 
ence; therefore, data from such males should be excluded from learning and memory analysis (see Step 16). In contrast, courtship suppression toward immature males can be reproduced without the male's performance of courtship during training. Exposure to the pheromone extract of immature males alone can induce behavioral modification (Gailey et al. 1982). For such a pheromone experiment, a double-layered courtship chamber with a mesh barrier can be used (see Measurement of Courtship Behavior in Drosophila melanogaster; Ejima and Griffith 2007). Another feature that separates courtship habituation from associative courtship conditioning is sensitivity to a dishabituation stimulus. The courtship suppression toward immature males can be dishabituated (e.g., by vortexing for $1 \mathrm{~min}$ ), whereas the courtship memory produced by conditioning using female targets is resistant to such mechanical disturbance (Ejima et al. 2005).

The persistence of the courtship modification largely depends on the experimental conditions during training. In a small chamber the male is continuously exposed to the unconditioned stimulus (US) and conditioned stimulus (CS) cues during training (for review, see Griffith and Ejima 2009), somewhat analogous to a "massed" training procedure. This type of small chamber training produces memory that lasts for hours. If training is carried out in a large chamber that allows relatively frequent breaks between bouts of courtship (a "spaced" training paradigm), memory can last for days (McBride et al. 1999). Lighting conditions are also critical for courtship experiments: Because visual cues are strong positive cues for male courtship, their availability can sometimes mask subtle changes in sexual motivation of the trained male or in changes in the salience of nonvisual cues (Joiner and Griffith 2000). Dim red light with wavelength $>700 \mathrm{~nm}$ can be used to substantially reduce visual information during the assay and increase emphasis on nonvisual cues. The effects of these and other sensory input on courtship are discussed in Measurement of Courtship Behavior in Drosophila melanogaster (Ejima and Griffith 2007).

\section{REFERENCES}

Ackerman SL, Siegel RW. 1986. Chemically reinforced conditioned courtship in Drosophila: Responses of wild-type and the dunce, amnesiac and don giovanni mutants. J Neurogenet 3: 111-123.

Ejima A, Griffith LC. 2007. Measurement of courtship behavior in Drosophila melanogaster. Cold Spring Harb Protoc doi: $10.1101 /$ pdb.prot.4847.

Ejima A, Smith BP, Lucas C, Levine JD, Griffith C. 2005. Sequential learning of pheromonal cues modulates memory consolidation in trainer-specific associative courtship conditioning. Curr Biol 15: 194-206.

Gailey DA, Jackson FR, Siegel RW. 1982. Male courtship in Drosophila: The conditioned response to immature males and its genetic con- trol. Genetics 102: 771-782.

Griffith LC, Ejima A. 2009. Courtship learning in Drosophila melanogaster: Diverse plasticity of a reproductive behavior. Learn Mem 16: 743-750.

Joiner MA, Griffith LC. 2000. Visual input regulates circuit configuration in courtship conditioning of Drosophila melanogaster. Learn Mem 7: 32-42.

McBride SM, Giuliani G, Choi C, Krause P, Correale D, Watson K, Baker G, Siwicki KK. 1999. Mushroom body ablation impairs short-term memory and long-term memory of courtship conditioning in Drosophila melanogaster. Neuron 24: 967-977.

\section{RECIPES}

[NOTE: Recipes for reagents marked with the $<\mathbf{R}>$ symbol not listed below can be found online at http://www. cshprotocols.org/recipes.]

\section{Food media}

$600 \mathrm{~g}$ agar

$1950 \mathrm{~g}$ yeast

$4571 \mathrm{~g}$ cornmeal

$6300 \mathrm{~g}$ dextrose

$480 \mathrm{~g}$ NaKT (sodium potassium tartrate)

$<$ ! $>60 \mathrm{~g} \mathrm{CaCl}_{2}$

$<$ !>169 g Lexgard M

$60 \mathrm{~L} \mathrm{H}_{2} \mathrm{O}$

Dissolve Lexgard $\mathrm{M}$ in ethanol $(10 \%, \mathrm{w} / \mathrm{v})$. Add all reagents to $\mathrm{H}_{2} \mathrm{O}$ and mix thoroughly. Autoclave and distribute into individual test tubes. 


\section{CAUTIONS}

[NOTE: For reagents marked with the $<$ ! $>$ symbol not listed below, please consult the manufacturer's Material Safety Data Sheet for further information.]

\section{$\mathrm{CaCl}_{2}$ (Calcium chloride)}

$\mathrm{CaCl}_{2}$ (Calcium chloride) is hygroscopic and may cause cardiac disturbances. It may be harmful by inhalation, ingestion, or skin absorption. Do not breathe the dust. Wear appropriate gloves and safety goggles.

\section{$\mathrm{CO}_{2}$ (Carbon dioxide; Dry ice)}

$\mathrm{CO}_{2}$ (carbon dioxide; dry ice) in all forms may be fatal by inhalation, ingestion, or skin absorption. In high concentrations, it can paralyze the respiratory center and cause suffocation. Use only in well-ventilated areas. In the form of dry ice, contact with carbon dioxide can also cause frostbite. Do not place large quantities of dry ice in enclosed areas such as cold rooms. Wear appropriate gloves and safety goggles.

\section{Ether (Diethyl ether, $\mathrm{Et}_{2} \mathrm{O},\left[\mathrm{C}_{2} \mathrm{H}_{5}\right]_{2} \mathrm{O}$ )}

Ether (diethyl ether, $\mathrm{Et}_{2} \mathrm{O},\left[\mathrm{C}_{2} \mathrm{H}_{5}\right]_{2} \mathrm{O}$ ) is extremely volatile and flammable. It is irritating to the eyes, mucous membranes, and skin. It is also a CNS depressant with anesthetic effects. It may be harmful by inhalation, ingestion, or skin absorption. Avoid breathing the vapors. Wear appropriate gloves and safety glasses. Always use in a chemical fume hood. Explosive peroxides can form during storage or on exposure to air or direct sunlight. Keep away from heat, sparks, and open flame.

\section{Lexgard (Tegosept, methyl paraben, Nipagin)}

Lexgard (Tegosept, methyl paraben, Nipagin) is an irritant and may be harmful by inhalation, ingestion, or skin absorption. Wear appropriate gloves and safety glasses. 


\section{Assay for Courtship Suppression in Drosophila}

Aki Ejima and Leslie C. Griffith

Cold Spring Harb Protoc; doi: 10.1101/pdb.prot5575

\begin{tabular}{|c|c|}
\hline $\begin{array}{r}\text { Email Alerting } \\
\text { Service }\end{array}$ & Receive free email alerts when new articles cite this article - click here. \\
\hline $\begin{array}{l}\text { Subject } \\
\text { Categories }\end{array}$ & $\begin{array}{l}\text { Browse articles on similar topics from Cold Spring Harbor Protocols. } \\
\text { Behavioral Assays (99 articles) } \\
\text { Drosophila (272 articles) } \\
\text { Genetics, general (375 articles) } \\
\text { Laboratory Organisms, general (924 articles) } \\
\text { Neuroscience, general (357 articles) }\end{array}$ \\
\hline
\end{tabular}

